\title{
Profile of people with hypertension in Nairobi's slums: a descriptive study
}

\author{
Annelieke Hulzebosch ${ }^{1 *}$, Steven van de Vijver ${ }^{2,3}$, Samuel O. Oti ${ }^{3}$, Thaddaeus Egondi ${ }^{3}$ and Catherine Kyobutungi ${ }^{3}$
}

\begin{abstract}
Background: Cardiovascular disease (CVD) is a rising health burden among the world's poor with hypertension as the main risk factor. In sub-Saharan Africa, hypertension is increasingly affecting the urban population of which a substantial part lives in slums. This study aims to give insight into the profile of patients with hypertension living in slums of Nairobi, Kenya.

Methods: Sociodemographic and anthropometric data as well as clinical measurements including BP from 440 adults with hypertension aged 35 years and above living in Korogocho, a slum on the eastern side of Nairobi, Kenya, will be collected at baseline and at the first clinic visit.

Conclusion: The study population showed high prevalence of overweight and abdominal obesity as well as behavioral risk factors such as smoking, alcohol and a low vegetable and fruit intake. Furthermore, the majority of hypertensive patients do not take anti-hypertensive medication and the ones who do show little adherence.
\end{abstract}

Trial registration: Current controlled trials ISRCTN84424579.

Keywords: Hypertension, Slums, Sub-Saharan Africa, Overweight, Awareness, Drug coherence, Diabetes

\section{Background}

In 2000, nearly one billion of the world's population (over $25 \%$ at that time) had hypertension and this is expected to increase to almost $30 \%$ by 2025 [1]. Hypertension is the single most important risk factor for cardiovascular disease (CVD) [2, 3], the leading cause of death worldwide [3]. The World Health Organization estimated that CVD caused 13.4 million deaths in 2008, $80 \%$ of which occurred in developing countries $[4,5]$.

In sub-Saharan Africa (SSA), the epidemiological transition of diseases causing mortality from predominantly infectious diseases to non-communicable diseases (NCDs) like CVD is attributable largely to urbanization and industrialization [6]. The changes in lifestyle associated with this transition may lead to an increase in the occurrence of behavioral risk factors for CVD such as smoking, physical inactivity and an unhealthy diet. Increases in mean blood pressure (BP) have already been observed in SSA. In Kenya for example, the average systolic BP has risen from 127 in 1990 to $132 \mathrm{mmHg}$ in 2010 [7]. The

\footnotetext{
* Correspondence: anneliekehulzebosch@gmail.com

'Utrecht University, Ameland 1953524 AN, Utrecht, Netherlands

Full list of author information is available at the end of the article
}

prevalence of risk factors for CVD including hypertension is higher in urban than in rural areas $[8,9]$. It is expected that the urban population in Africa will increase by half in the next decades [10]. The urban population growth rate in Nairobi is $4.2 \%$, which is almost double the national growth rate of $2.4 \%$ [11]. Nearly $60 \%$ of the urban population in Nairobi lives in slums or slum-like conditions $[12,13]$. Slums are typically characterized by poor living conditions with limited access to quality healthcare [14]. The prevalence of risk factors for CVD is high in slums and the associated psychosocial burden of insecurity, violence and stress may cause an increased risk of CVD [15]. Therefore a large part of the CVD burden is on the urban poor, who may not have the financial resources or adequate health literacy to adopt healthier lifestyles to adopt preventive measures [16, 17].

Despite the large extent of the epidemic, there is a paucity of documentation on the profile of the growing group of urban poor people with hypertension in SSA. This descriptive study aims to give an insight into patients with hypertension in a slum in Nairobi. What are the characteristics of these people, their behavioral risk factors, medication use and comorbidities? 


\section{Methods}

\section{Study population and design}

The study was conducted in Korogocho, a large slum with approximately 72.000 residents in the outskirts of Nairobi, the capital city of Kenya. Korogocho is under surveillance as part of the Nairobi Urban Health and Demographic Surveillance System (NUHDSS). The NUHDSS is operated by the African Population and Health Research Center (APHRC), a regional research institution. Details of the operation of the NUHDSS have been published elsewhere [18].

Data from 440 individuals were collected between August 2012 and December 2013. The data-collection was done as part of a published cross-sectional survey called the SCALE UP project, which aimed to develop and introduce a cost-effective and scalable CVD prevention model among the urban poor [19]. The goal of the prevention model was to raise awareness of hypertension through mass screening of adult slum residents aged 35 years and older, to improve access to treatment and promote adherence to medication. This paper examines adults with hypertension detected through the SCALE UP study and referred to local primary health care facility for treatment and follow-up.

As part of the SCALE UP study, 40 interviewers and 40 community health workers (CHWs) trained and employed by APHRC performed household visits to screen participants for hypertension and risk factors. The interviewers administered the questionnaire in the local lingua franca - Kiswahili. This questionnaire was adapted from the WHO STEPs instrument and gathered information on demographics, education level, wealth and behavioral risk factors [20]. After the questionnaires were administered, the $\mathrm{CHW}$ s proceeded to take anthropometric measurements from all consenting participants including weight, height and waist circumference. While an elevated BMI is a well-established contributor to the etiology of CVD, studies have shown that abdominal obesity are more closely related to CVD morbidity and mortality than BMI alone [20]. Therefore, both measures have been included in this study. All measurements were taken in line with the STEPS protocol [21]. Stage one hypertension was defined as mean systolic blood pressure (SBP) at least $140 \mathrm{mmHg}$ or diastolic blood pressure (DBP) at least $90 \mathrm{mmHg}$, or self-reported previous diagnosis and current use of antihypertensive medication. Stage two hypertension was defined as mean SBP at least $160 \mathrm{mmHg}$ or DBP at least $100 \mathrm{mmHg}$ [2]. Mean arterial pressure was diastolic pressure plus one third of systolic BP. Treatment control was defined as BP of less than 140/90 $\mathrm{mmHg}$ for those participants currently taking anti-hypertensive medication. Participants with hypertension detected through the household visits were referred to a local primary health clinic known as Provide International, where they were interviewed again trained community health workers for risk re-assessment purposes. Participants were then assessed by clinical officers who confirmed their diagnoses. Those confirmed with hypertension and who provided informed consent became subjects of this current study. Overall, three levels of information were collected from participants; a questionnaire to gather information about history of hypertension and comorbidities, basic anthropometric and clinical measurements and a random blood glucose test.

Average monthly income was categorized into four groups, where 1000 KES equals \$11.10. Alcohol consumption was classified as low $(<1$ standard unit of alcohol per week), moderate (1-14 per week for males and 1-7 per week for females) and high (>14 per week for males and $>7$ per week for females). Adequate physical activity was defined as more than 150 min of moderate intensity activity or more than $75 \mathrm{~min}$ vigorous intensity activity per week [22, 23]. Adequate fruit and vegetable intake was defined as 5 servings of fruit or vegetables per day, using a serving size of $80 \mathrm{~g}$ and the daily minimum of $400 \mathrm{~g}$ [24-26].

Field supervisors verified the data quality. Questionnaires that did not meet the standards were corrected in the field. The completed questionnaires were then transported to a central location for double-data entry using MySQL data entry screens with Microsoft Access database backend.

\section{Statistical analysis}

Frequency distributions of sociodemographic, behavioral and clinical characteristics of study subjects by gender were examined. Continuous variables were expressed as means with standard errors of means. Categorical variables were expressed as numbers with percentages. For those variables where over $10 \%$ of results were missing, 'missing' was listed as a category and thus percentages adjusted accordingly.

Association of the study participants' sociodemographic and behavioral risk factors by sex was conducted using chi square tests for significance. A value of $P<0.05$ was considered significant. All analyses were performed with STATA 12 (Stata Corp, College Station, Texas).

\section{Ethical approval}

The study protocol was approved by the Kenya Medical Research Institute (KEMRI)/National Ethical Review Committee (NON-SSC Protocol No.339).

\section{Results}

There were 256 women (58 \%) and 184 men (42\%) in the study. It is noteworthy that although a total of 976 were referred from the screening during the household 
visits, only 440 (46\%) who attended the local primary clinic subsequently as at the time of conducting this analysis were included.

Table 1 shows sociodemographic variables as well as behavioral risk factors. Men were significantly more educated than women and reported a higher average monthly income. Gender disparities were also observed in CVD risk factors, significantly more men smoked and consumed alcohol. There was no significant difference in reported physical activity and daily fruit and vegetable intake between genders. Overall individuals demonstrated a high level of physical activity but a very low

Table 1 Description of study participants by gender

\begin{tabular}{|c|c|c|c|c|c|c|c|c|c|}
\hline & \multicolumn{3}{|c|}{ All $(N=440)$} & \multicolumn{3}{|c|}{ Women $(N=256)$} & \multicolumn{3}{|c|}{ Men $(N=184)$} \\
\hline & $N$ & $\%$ & $95 \% \mathrm{Cl}$ & $N$ & $\%$ & $95 \% \mathrm{Cl}$ & $N$ & $\%$ & $95 \% \mathrm{Cl}$ \\
\hline \multicolumn{10}{|l|}{ Age (years) } \\
\hline$<45$ & 67 & 15.2 & $12.2-18.9$ & 41 & 16.0 & $12.0-21.1$ & 26 & 14.1 & $9.8-20.0$ \\
\hline $45-54$ & 154 & 35.0 & $30.7-39.6$ & 92 & 35.9 & $30.3-42.0$ & 62 & 33.7 & $27.2-40.9$ \\
\hline $55-64$ & 102 & 23.2 & $19.5-27.4$ & 51 & 19.9 & $15.5-25.3$ & 51 & 27.7 & $21.7-34.7$ \\
\hline $65-74$ & 78 & 17.7 & $14.4-21.6$ & 46 & 18.0 & $13.7-23.2$ & 32 & 17.4 & $12.6-23.6$ \\
\hline$\geq 75$ & 39 & 8.9 & $6.5-11.9$ & 26 & 10.2 & $7.0-14.5$ & 13 & 7.1 & $4.1-11.8$ \\
\hline \multicolumn{10}{|l|}{ Education $P<0,01$} \\
\hline Not finished primary school & 99 & 22.5 & & 66 & 25.8 & & 38 & 17.9 & \\
\hline Primary school & 171 & 38.9 & & 89 & 34.8 & & 82 & 44.6 & \\
\hline Secondary school and higher & 54 & 12.3 & & 16 & 6.3 & & 33 & 20.7 & \\
\hline Missing & 116 & 26.4 & & 85 & 33.2 & & 31 & 16.8 & \\
\hline \multicolumn{10}{|l|}{ Marital status $P<0,01$} \\
\hline Currently married & 269 & 61.3 & & 108 & 42.2 & & 161 & 88.0 & \\
\hline Never married & 30 & 6.8 & & 23 & 9.0 & & 7 & 3.8 & \\
\hline Widowed & 89 & 20.3 & & 80 & 31.3 & & 9 & 4.9 & \\
\hline Divorced/separated & 51 & 11.6 & & 45 & 17.6 & & 6 & 3.3 & \\
\hline \multicolumn{10}{|l|}{ Monthly income $P<0,01$} \\
\hline$<1000 \mathrm{KES}$ & 37 & 8.4 & & 29 & 11.3 & & 8 & 4.3 & \\
\hline 1000-4999 KES & 180 & 40.9 & & 119 & 46.5 & & 61 & 33.2 & \\
\hline 5000-9999 KES & 88 & 20.0 & & 31 & 12.1 & & 57 & 31.0 & \\
\hline$\geq 10000 \mathrm{KES}$ & 23 & 5.2 & & 5 & 2.0 & & 18 & 9.8 & \\
\hline Missing & 112 & 25.5 & & 72 & 28.1 & & 40 & 21.7 & \\
\hline \multicolumn{10}{|l|}{ Smoking status $P<0,01$} \\
\hline Current smoker & 37 & 8.4 & & 5 & 2.0 & & 32 & 17.5 & \\
\hline Previous smoker & 52 & 11.8 & & 18 & 7.0 & & 34 & 18.6 & \\
\hline Non-smoker & 350 & 79.7 & & 233 & 91.0 & & 117 & 63.9 & \\
\hline \multicolumn{10}{|l|}{ Alcohol consumption $P<0,01$} \\
\hline Low & 373 & 84.8 & & 237 & 92.6 & & 136 & 73.9 & \\
\hline Moderate & 30 & 6.8 & & 9 & 3.5 & & 21 & 11.4 & \\
\hline High & 37 & 8.4 & & 10 & 3.9 & & 27 & 14.7 & \\
\hline \multicolumn{10}{|l|}{ Physical activity $P=0.17$} \\
\hline Adequate & 401 & 91.8 & & 231 & 90.2 & & 170 & 93.9 & \\
\hline Inadequate & 36 & 8.2 & & 25 & 9.8 & & 11 & 6.1 & \\
\hline \multicolumn{10}{|c|}{ Daily fruit and vegetable intake $P=0.12$} \\
\hline Adequate & 76 & 17.3 & & 49 & 19.1 & & 27 & 14.7 & \\
\hline Inadequate & 299 & 68.0 & & 176 & 68.8 & & 123 & 66.8 & \\
\hline Missing & 65 & 14.8 & & 31 & 12.1 & & 34 & 18.5 & \\
\hline
\end{tabular}

Missing data: marital status 1, smoking status 1, physical activity 3. $P$ values for difference between genders derived using chi-squared test 
level of fruit and vegetable intake. As can be seen in Table 2, women had a higher BMI with $63 \%$ being overweight or obese. Men had a significantly larger waist circumference; almost half of them fit the criteria for abdominal obesity. The mean SBP was $149.2 \mathrm{mmHg}$ and the mean DBP was $95.2 \mathrm{mmHg}$. Women had a lower SBP than men. Over $40 \%$ of individuals fit the criteria for stage two hypertension. Table 3 shows the history of the participants' hypertension. Almost $70 \%$ were diagnosed in the last two years and $68 \%$ were diagnosed through a CHW visiting their home as part of the SCALE UP study. Only a quarter of the participants with hypertension ever used medication for their condition. Of the people who did, the majority $(78 \%)$ used the medication daily. The reported total costs of antihypertensive medication vary but over $75 \%$ spent more than 100 KES per month (about \$1.15). Treatment control was $53.8 \%$ (95 \% CI 34.7-73.0). Almost half of the medication users are currently not taking the medication anymore. Over a third of patients felt better and therefore believed they did not need the medication anymore, and almost half of the group reported they stopped using the anti-hypertensive drugs because they could no longer afford it. Overall $10 \%$ of the patients with hypertension were found to have diabetes. Only $6.8 \%$ reported to be aware of this condition prior to the CHW's home visit. Table 4 shows other reported comorbidities.

\section{Discussion}

This study describes the profile of hypertensive patients living in an informal settlement in Kenya. Compared to a recent population wide survey in Korogocho, our study participants smoke and consume more alcohol, and there is more obesity and less fruit and vegetable intake [15]. The differences could be explained by the type of study participants, our study only included hypertensive patients aged 35 years and older whereas the previous survey included a representative urban slum population of adults aged 18 years and older. So compared to a largely healthy population, patients with hypertension will expectedly show different characteristics. For example, the percentage of men in our study with abdominal obesity (48.6\%) is very high compared to the aforementioned survey in the same slum where only $11.6 \%$ of men fit the criteria.

Our study shows a higher rate of participants classifying as overweight or obese than a recent study on overweight in Korogocho that found overweight and obesity rates similar to that of a national survey; around $40 \%$ in women and $17 \%$ in men $[27,28]$. Studies from Tanzania and Cameroon show roughly the same gender disparities in prevalence of excess bodyweight $[29,30]$. In our study we have found overweight and obesity prevalence to be much higher at $63 \%$ and $34.8 \%$ for women and men respectively, but again, our study sample consists of selected individuals with known hypertension. The high proportion of overweight and obesity specifically among women could be explained by different socio-cultural factors such as gender specific patterns of work activities and cultural standards of physical attractiveness [31].

Currently the estimated prevalence of diabetes in Africa is $1-3 \%$ in rural areas and 5-6\% in urban SSA, but country reports have varied widely [32]. The

Table 2 Anthropometric and biochemical measurements

\begin{tabular}{|c|c|c|c|c|c|c|c|c|c|}
\hline & \multicolumn{3}{|c|}{ All $(N=440)$} & \multicolumn{3}{|c|}{ Women $(N=256)$} & \multicolumn{3}{|c|}{ Men $(N=184)$} \\
\hline & $N$ & $\%$ & $95 \% \mathrm{Cl}$ & $N$ & $\%$ & $95 \% \mathrm{Cl}$ & $N$ & $\%$ & $95 \% \mathrm{Cl}$ \\
\hline \multicolumn{10}{|l|}{ BMI $P<0.01$} \\
\hline Underweight $(<18.5)$ & 26 & 6.0 & $4.1-8.7$ & 13 & 5.2 & $3.0-8.8$ & 12 & 7.2 & $4.2-12.0$ \\
\hline Normal weight (18.5-24.9) & 184 & 42.8 & $38.2-47.5$ & 79 & 31.7 & $26.2-37.8$ & 105 & 58 & $50.7-65.0$ \\
\hline Overweight (25-29.9) & 130 & 30.2 & $26.1-34.8$ & 88 & 35.3 & $29.6-41.5$ & 42 & 23.2 & $17.6-29.9$ \\
\hline Obese $(\geq 30)$ & 90 & 20.9 & $17.3-25.1$ & 69 & 27.7 & $22.5-33.6$ & 21 & 11.6 & $7.7-17.2$ \\
\hline \multicolumn{10}{|c|}{ Waist circumference $P<0.01$} \\
\hline Normal & 293 & 67.8 & $63.3-72.1$ & 200 & 79.7 & $74.2-84.2$ & 93 & 51.4 & $44.1-58.6$ \\
\hline Abdominal obesity & 139 & 32.2 & $27.9-36.7$ & 51 & 20.3 & $15.8-25.8$ & 88 & 48.6 & $41.4-55.9$ \\
\hline Diabetes mellitus & 41 & 10.0 & $7.5-13.4$ & 26 & 11.0 & $7.6-15.6$ & 15 & 8.8 & $5.3-14.1$ \\
\hline \multicolumn{10}{|l|}{ Blood pressure } \\
\hline Mean SBP & 149.2 & \multicolumn{2}{|c|}{$147.1-151.3$} & 147.3 & \multicolumn{2}{|c|}{$144.4-150.1$} & 151.9 & \multicolumn{2}{|c|}{$148.7-155.1$} \\
\hline Mean DBP & 95.2 & \multicolumn{2}{|c|}{$94.0-96.4$} & 95.5 & \multicolumn{2}{|c|}{$94.0-97.0$} & 94.8 & \multicolumn{2}{|c|}{$92.9-96.7$} \\
\hline Mean arterial pressure & 145.0 & \multicolumn{2}{|c|}{$143.2-146.7$} & 144.6 & \multicolumn{2}{|c|}{$142.4-146.9$} & 145.4 & \multicolumn{2}{|c|}{$142.7-148.2$} \\
\hline Stage two hypertension & 177 & 40.2 & $35.7-44.9$ & 100 & 39.1 & $33.3-45.2$ & 77 & 41.8 & $34.9-49.1$ \\
\hline
\end{tabular}


Table 3 History of hypertension

\begin{tabular}{|c|c|c|c|}
\hline & \multicolumn{3}{|c|}{ All $(N=440)$} \\
\hline & $N$ & $\%$ & $95 \% \mathrm{Cl}$ \\
\hline \multicolumn{4}{|l|}{ Time since diagnosis (years) } \\
\hline$<1$ & 14 & 3.2 & $1.9-5.3$ \\
\hline $1-2$ & 285 & 64.8 & $60.2-69.1$ \\
\hline $2-5$ & 56 & 12.7 & $9.9-16.2$ \\
\hline $5-10$ & 39 & 8.9 & $6.5-11.9$ \\
\hline$>10$ & 46 & 10.5 & $7.9-13.7$ \\
\hline \multicolumn{4}{|l|}{ Place of diagnosis } \\
\hline CHW visit & 298 & 68.5 & \\
\hline Private healthcare facility & 92 & 21.1 & \\
\hline Public healthcare facility & 45 & 10.3 & \\
\hline \multicolumn{4}{|l|}{ Ever used anti-hypertensive drugs } \\
\hline No & 336 & 76.5 & \\
\hline Yes & 103 & 23.5 & \\
\hline \multicolumn{4}{|l|}{ Medication use } \\
\hline Daily & 80 & 77.7 & \\
\hline On most days & 10 & 9.7 & \\
\hline On some days & 7 & 6.8 & \\
\hline When I felt bad & 6 & 5.8 & \\
\hline \multicolumn{4}{|c|}{ Total cost of anti-hypertensive drugs per month } \\
\hline No cost, free & 13 & 12.6 & \\
\hline$<100$ KES & 11 & 10.7 & \\
\hline 100-299 KES & 30 & 29.1 & \\
\hline 300-499 KES & 28 & 27.2 & \\
\hline$\geq 500 \mathrm{KES}$ & 21 & 20.4 & \\
\hline \multicolumn{4}{|l|}{ Currently taking the anti-hypertensive drugs } \\
\hline Yes & 54 & 52.4 & \\
\hline No & 49 & 47.6 & \\
\hline \multicolumn{4}{|c|}{ Reason for stopping the anti-hypertensive drugs } \\
\hline Could no longer afford the medication & 25 & 51.0 & \\
\hline I felt better, did not need further treatment & 15 & 30.6 & \\
\hline Other & 9 & 18.4 & \\
\hline
\end{tabular}

Missing data: place of diagnosis 2, ever used anti-hypertensive drugs 1

prevalence of diabetes is likely to be higher in people with hypertension than at a population level. A study in Nigeria found that $4.6 \%$ of hypertensive patients had diabetes [33]. Our study found this number to be over twice as high (10\%).

Table 4 Self reported history of comorbidities

\begin{tabular}{|c|c|c|c|c|c|c|c|c|c|}
\hline & \multicolumn{3}{|c|}{ All $(N=440)$} & \multicolumn{3}{|c|}{ Women $(N=256)$} & \multicolumn{3}{|c|}{ Men $(N=184)$} \\
\hline & $\bar{N}$ & $\%$ & $95 \% \mathrm{Cl}$ & $\bar{N}$ & $\%$ & $95 \% \mathrm{Cl}$ & $\bar{N}$ & $\%$ & $95 \% \mathrm{Cl}$ \\
\hline Diabetes & 30 & 6.8 & $4.8-9.6$ & 23 & 9.0 & $6.0-13.2$ & 7 & 3.8 & $1.8-7.8$ \\
\hline Stroke & 7 & 1.6 & $0.8-3.3$ & 3 & 1.2 & $0.4-3.6$ & 4 & 2.2 & $0.8-5.7$ \\
\hline Angina & 5 & 1.1 & $0.5-2.7$ & 3 & 1.2 & $0.4-3.6$ & 2 & 1.1 & $0.3-4.3$ \\
\hline
\end{tabular}

Missing data: diabetes, stroke and angina 1
Evidence shows that CVD risk factors occur predominantly in clusters [34]. People with diabetes are more likely to also suffer from hypertension and/or dyslipidemia. This is likely to also occur in our study population, given the high obesity and abdominal obesity prevalence suggesting a strong influence of metabolic syndrome in this population.

\section{Recommendations}

There should be more attention paid to the large and growing epidemic of CVD in SSA and a joint effort to stop it. Policy makers and health providers need to work together. This paper shows that weight control is an important priority in both men and women and that cost of medication plays a major role in compliance. A possible solution could be a community health insurance or drug revolving funds [35].

Levels of health literacy have to increase in order for people to change their lifestyle, get diagnosed or comply with therapy. The WHO developed"best buys" for policy makers to tackle the main non-communicable diseases. These "best buys" state that providing counseling and multi-drug therapy for people with high risk of CVD can be used together with population-level interventions like taxes on tobacco to decentivize smoking, legislation to promote reduced salt foods for mitigating hypertension and improving the public awareness of the role of diet and physical activity on health through mass media. These interventions have been found to have a significant impact and be highly cost-effective. The implementation of these initiatives in a slum context is urgently needed.

\section{Strengths and limitations of the study}

To the best of our knowledge this is the first study on the profile of hypertensive patients in a Kenyan urban informal settlement. Our study adds to the limited body of evidence on hypertension in Kenya and gives an insight to the patients' living standards and their rationale. This study has several limitations. One is the self-reporting of patients' wealth, education, behavioral risk factors (smoking, alcohol misuse, diet and physical activity), comorbidities and use of medication. Self-reporting can lead to inaccurate reporting due to lack of awareness, misinterpretation of questions, or concern for judgement and affect our study conclusions. Additionally, our study did not capture over $50 \%$ of patients during household screening visits or clinic visits. Follow up studies in this sub-population of patients would be very helpful. Despite these limitations, this study provides important data regarding the profile of patients with hypertension in an African urban slum. 


\section{Conclusions}

The study population showed high prevalence of overweight and abdominal obesity as well as behavioral risk factors such as smoking, alcohol and a low vegetable and fruit intake. Furthermore, the vast majority of hypertensive patients do not take anti-hypertensive medication and the ones who do show little adherence.

\section{Abbreviations}

CVD: Cardiovascular disease; SSA: Sub-Saharan Africa; NCDs: Non-communicable diseases; BP: Blood pressure; NUHDSS: Nairobi Urban Health and Demographic Surveillance System; CHW: Community health worker; SBP: Systolic blood pressure; DBP: Diastolic blood pressure; KEMRI: Kenya medical research institute.

\section{Competing interests}

The authors declare that they have no competing interests.

\section{Authors' contributions}

SV and SO organized data collection. AH participated in the design of the study, performed the statistical analysis and drafted the manuscript. TE performed statistical analysis and participated in study design. CK, SV and SO conceived of the study, participated in its design and coordination and helped to draft the manuscript. All authors read and approved the final manuscript.

\section{Acknowledgments}

We would like to thank the following people and organizations for their support in this study: the staff of APHRC for their support through drivers, field interviewers, research assistants and statisticians; staff of AIGHD for their practical and scientific support. We thank Dr. Tuinenburg of the UMC Utrecht for his general advice. Financial support for the execution of the project was from the Academic Medical Center (AMC) Foundation Amsterdam. This work was also made possible by core funding for APHRC from The William and Flora Hewlett Foundation (grant no. 2009-40510), the Swedish International Cooperation Agency (SIDA) (grant no. 2011-001578) and the Rockefeller Foundation (grant no. 2009SCG302).

\section{Author details}

${ }^{1}$ Utrecht University, Ameland 1953524 AN, Utrecht, Netherlands.

${ }^{2}$ Department of Global Health, Academic Medical Center, University of Amsterdam, Nairobi, Kenya. ${ }^{3}$ African Population and Health Research Center, Nairobi, Kenya.

Received: 18 January 2015 Accepted: 11 June 2015

Published online: 27 June 2015

\section{References}

1. Kearney PM, Whelton M, Reynolds K, Muntner P, Whelton PK, He J. Global burden of hypertension: analysis of worldwide data. Lancet. 2005;365:217-23.

2. Chobanian AV, Bakris GL, Black HR, Cushman WC, Green LA, Izzo JL, et al. Seventh report of the joint national committee on prevention, detection, evaluation, and treatment of high blood pressure. Hypertension. 2003:42:1206-52.

3. Lopez AD, Mathers CD, Ezzati M, Jamison DT, Murray CJ. Global and regional burden of disease and risk factors, 2001: systematic analysis of population health data. Lancet. 2006;367:1747-57.

4. World Health Organization. The global burden of disease: 2004 update. Geneva 2008. Available at: http://www.who.int/healthinfo/global_burden_ disease/2004_report_update/en/ [Accessed 12 November 2014]

5. Gaziano TA, Opie LH, Weinstein MC. Cardiovascular disease prevention with a multidrug regimen in the developing world: a cost-effectiveness analysis. Lancet. 2006;368:679-86.

6. Godfrey R, Julien M. Urbanisation and health. Clin Med J R Coll Phys. 2005:5:137-41.

7. Imperial College London. Global burden of metabolic risk factors of chronic diseases. 2012. Available at: http://www.imperial.ac.uk/medicine/ globalmetabolics/. [Accessed 12 November 2014]
8. Cardiovascular Diseases in the African Region: Current Situation and Perspectives, WHO, 17 June 2005. Available at: http://www.afro.who.int/ index.php?option=com_docman\&task=doc_download\&gid=2305[Accessed 24 March 2014]

9. Fuentes R, Ilmaniemi N, Laurikainen E, Tuomilehto J, Nissinen A. Hypertension in developing economies: a review of population-based studies carried out from 1980 to 1998. J Hypertens. 2000;18:521-9.

10. United Nations. World Urbanization Prospects The 2007 Revision Highlights. New York: ESA/P/WP/2; 2007. p. 883.

11. Kenya National Bureau of Statistics (KNBS) and ICF Macro. 2010 Kenya Demographic and Health Survey 2008-09. Calverton, Maryland: KNBS and ICF Macro. Available at: \http://dhsprogram.com/pubs/pdf/FR229/FR229.pdf [accessed 24 december 2014]

12. UN Habitat, the State of African Cities: Nairobi, available at: http://www.ruaf.org/sites/default/files/Habitat\%20state\%20of\%20African\% 20cities.pdf [accessed 12 March 2014]

13. United Nations Human Settlements Programme (UN-HABITAT). Nairobi urban sector profile. Nairobi: UN-HABITAT; 2006.

14. Lamba D. The forgotten half; environmental health in Nairobi's poverty areas. Environ Urban. 1994;6(1):164-73.

15. Van de Vijver SJM, Oti SO, Agyemang C, Gomez GB, Kyobutungi C. Prevalence, awareness, treatment and control of hypertension among slum dwellers in Nairobi Kenya. J Hypertens. 2013;31(5):1018-24.

16. Popkin BM, Gordon-Larsen P. The nutrition transition: worldwide obesity dynamics and their determinants. Int J Obes Relat Metab Disord. 2004;28(3):S2-9.

17. Nyaruhucha CNM, Achen JH, Msuya JM, Shayo NB, Kulwa KBM. Prevalence and awareness of obesity among people of different age groups in educational institutions in Morogoro Tanzania. East Afr Med J. 2003:80:68-72.

18. Emina J, Beguy D, Zulu EM, Ezeh AC, Muindi K, Elung'ata P, et al. Monitoring of health and demographic outcomes in poor urban settlements: evidence from the Nairobi Urban Health and Demographic Surveillance System. J Urban Health: Bull N Y Acad Med. 2011;88(2):S200-18.

19. Oti SO, van de Vijver SJM, Kyobutungi C, Gomez GB, Agyemang C, Van Charante EPM, et al. A community-based intervention for primary prevention of cardiovascular diseases in the slums of Nairobi: the SCALE UP study protocol for a prospective quasi-experimental community-based trial. Trials. 2013;14:409.

20. Lee CMY, Huxley RR, Wildman RP, Woodward M. Indices of abdominal obesity are better discriminators of cardiovascular risk factors than BMI: a meta-analysis. J Clin Epidemiol. 2008;61(7):646-53.

21. The WHO STEPwise approach to chronic disease risk factor surveillance, version 3.0, June 2013, available at: http://www.who.int/chp/steps/ instrument/STEPS_Instrument_V3.0.pdf?ua=1 [accessed on 15 April 2014]

22. World Health Organization, Global Strategy on Diet, Physical Activity and Health: Intensity of physical activity, available at: http://www.who.int/ dietphysicalactivity/physical_activity_intensity/en/ [accessed 24 March 2014]

23. World Health Organization, Physical activity recommendations. http://www.who.int/dietphysicalactivity/physical-activity-recommendations18-64years.pdf?ua=1 [accessed 24 March 2014]

24. Agudo A. World health organization background paper: measuring intake of fruit and vegetables, available at: http://www.who.int/dietphysicalactivity/ publications/f\&v_intake_measurement.pdf [accessed on 25 March 2014]

25. World Health Organization technical report series: diet, nutrition and the prevention of chronic diseases, available at: http://whqlibdoc.who.int/trs/ who trs 916.pdf [accessed on 25 March 2014]

26. NHS rough guide: fruit and vegetable portion size, available at: http:// www.nhs.uk/livewell/5aday/documents/downloads/5aday_portion_ guide.pdf [accessed on 25 March 2014]

27. Ettarh R, Van de Vijver S, Oti S, Kyobutungi C. Overweight, obesity, and perception of body image among slum residents in Nairobi, Kenya, 2008-2009. Prev Chronic Dis. 2013;10:E212.

28. Ziraba AK, Fotso JC, Ochako R. Overweight and obesity in urban Africa: a problem of the rich or the poor? BMC Public Health. 2009;9:465. doi:10.1186/1471-2458-9-465.

29. Njelekela MA, Mpembeni R, Muhihi A, Mligiliche NL, Spiegelman D,

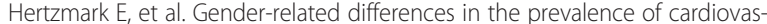
cular disease risk factors and their correlates in urban Tanzania. BMC Cardiovasc Disord. 2009:9:30.

30. Sobngwi E, Mbanya J-CN, Unwin NC, Kengne AP, Fezeu L, Minkoulou EM, et al. Physical activity and its relationship with obesity, hypertension and 
diabetes in urban and rural Cameroon. Int J Obes Relat Metab Disord. 2002;26:1009-16

31. Ayah R, Joshi MD, Wanjiru R, Njau EK, Otieno CF, Njeru EK, et al. A population-based survey of prevalence of diabetes and correlates in an urban slum community in Nairobi Kenya. BMC Public Health. 2013;13:371.

32. Siervo M, Grey P, Nyan OA, Prentice AM. A pilot study on body image, attractiveness and body size in Gambians living in an urban community. Eat Weight Disord. 2006;11:100-9.

33. Mbanya JCN, Motala AA, Sobngwi E, Assah FK, Enoru ST. Diabetes in sub-Saharan Africa. Lancet. 2010;375(9733):2254-66.

34. Ogunleye OO, Ogundele SO, Akinyemi JO, Ogbera O. Clustering of hypertension, diabetes mellitus and dyslipidemia in a Nigerian population: a cross sectional study. Afr J Med Med Sci. 2012:41(2):191-5.

35. Hendriks ME, Wit FW, Akande TM, Kramer B, Osagbemi GK, Tanovic Z, et al. Effect of health insurance and facility quality improvement on blood pressure in adults with hypertension in Nigeria: a population-based study. JAMA Intern Med. 2014;174(4):555-63.

\section{Submit your next manuscript to BioMed Central and take full advantage of:}

- Convenient online submission

- Thorough peer review

- No space constraints or color figure charges

- Immediate publication on acceptance

- Inclusion in PubMed, CAS, Scopus and Google Scholar

- Research which is freely available for redistribution 